\title{
Anisotropy of the Elastic Constants in SmC* Liquid Crystal Films
}

\author{
K. J. Kidney \\ I. W. Stewart \\ G. McKay \\ Department of Mathematics, University of Strathclyde, Glasgow, \\ Scotland, UK
}

We consider a film of SmC* liquid crystal with its layers parallel to an applied electric field. The liquid crystal is assumed to align uniformly under the influence of the field, except along domain walls. By employing a nonlinear continuum theory for smectic liquid crystals we can examine the width of domain walls perpendicular and parallel to the field. We obtain predictions for the wall widths based on the elastic constants and dielectric properties of the $\mathrm{SmC}^{*}$ liquid crystal. Exact analytical expressions for the wall widths are obtained by assuming series expansions in the elastic anisotropy and dielectric parameters.

Keywords: dielectric; domain walls; smectic liquid crystals

\section{INTRODUCTION}

It is known that surface tension can induce a smectic film at the surface of an isotropic liquid crystal droplet, see for example Swanson et al. [1]. The molecules within the smectic layers can also orientate uniformly under the influence of an applied electric field in the plane of the smectic layers, except possibly along domain walls. Candel and Galerne [2] examined the widths of these walls in smectic $\mathrm{O}(\mathrm{SmO})$ liquid crystals. In particular, they compared the ratio of theoretical wall widths (obtained via an approximation of the governing equations for director twist) with experimental data in order to derive information about the influence of anisotropy in the elastic constants upon the induced $\mathrm{SmO}$ film.

Address correspondence to G. McKay, Department of Mathematics, University of Strathclyde, 26 Richmond Street, Glasgow, G1 1 XH, Scotland, UK. E-mail: gmck@maths.strath.ac.uk 
Here we adopt a similar approach to Candel and Galerne [2], except that we consider domain walls within ferroelectric $\mathrm{SmC}^{*}$ liquid crystal films. We determine an analytical expression for the ratio of wall widths that is dependent upon both dielectric anisotropy and the anisotropy of the twist smectic elastic constants, as employed in the theory introduced by Leslie et al. [3]. Numerical predictions for the wall widths can be determined via analysis of the director equations followed by numerical quadrature, or through power series expansions in the anisotropy and dielectric parameters.

\section{MODEL}

We consider a film of $\mathrm{SmC}^{*}$ liquid crystal with its layers perpendicular to the $z$-direction, as shown in Figure 1 , and a domain wall in the $x$-direction. An electric field is applied in a direction parallel to the smectic layers which may be either parallel or perpendicular to the domain wall. We model the behaviour of the liquid crystal under the influence of this field by employing the continuum theory for smectics contained in [3]. The $\mathrm{SmC}^{*}$ liquid crystal is assumed to be incompressible and in the isothermal state. The smectic cone angle $\theta$ is therefore fixed and the interlayer spacing is constant. The (unit) director representing the average molecular axis may be written as $\boldsymbol{n}=\boldsymbol{a} \cos \theta+\boldsymbol{c} \sin \theta$ where $\boldsymbol{a}$ is the unit normal vector for the smectic layer and $c=(\cos \phi(x), \sin \phi(x), 0)$ is the unit orthogonal projection of $\boldsymbol{n}$ onto the smectic layers where $\phi(x)$ is the orientation angle of the vector $c$ within the $x y$-plane. As we will see shortly, it is also convenient to introduce unit vector $\boldsymbol{b}=\boldsymbol{a} \times \boldsymbol{c}$. Knowledge of the orientation of $\boldsymbol{c}$ completely determines the sample alignment when $\boldsymbol{a}$ is fixed.
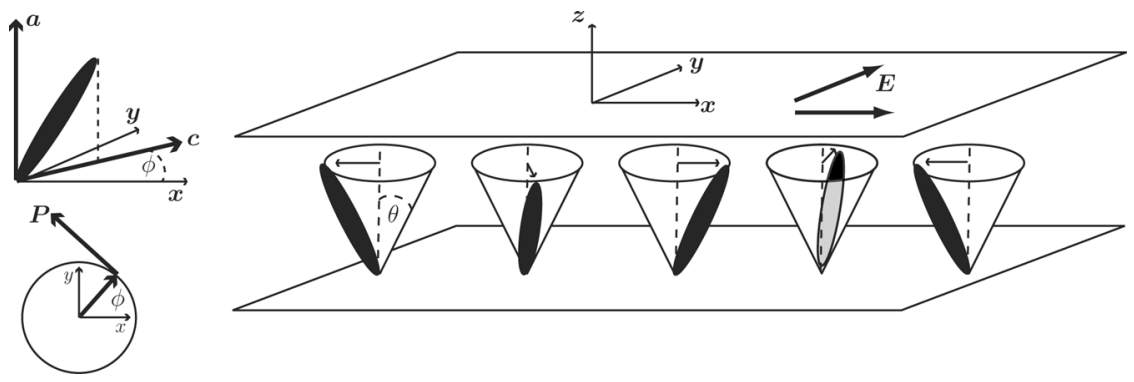

FIGURE 1 Description of vectors $\boldsymbol{a}, \boldsymbol{c}$ and the smectic film with its layer normals parallel to the $z$-direction. We consider electric fields applied in the $x$ and $y$-directions. 
The energy density for smectic C liquid crystals presented by Leslie et al. [3] can be described in terms of five basic deformations and involves nine elastic constants. However, for the geometry and director configuration considered here, the $\mathrm{SmC}^{*}$ elastic energy density, $f_{e}$, simplifies to

$$
f_{e}=\frac{1}{2} B_{1}(\nabla \cdot \boldsymbol{b})^{2}+\frac{1}{2} B_{2}(\nabla \cdot \boldsymbol{c})^{2}=\frac{1}{2}\left(B_{1} \cos ^{2} \phi+B_{2} \sin ^{2} \phi\right)\left(\frac{d \phi}{d x}\right)^{2} .
$$

The positive elastic constants $B_{1}$ and $B_{2}$ are related to twist deformations of the director within the smectic plane (see Stewart [4]). Our aim is to examine and obtain measurements for the anisotropy of these constants. With this in mind, we introduce a dimensionless measure of anisotropy, $\xi$, by assuming $B_{1}=B(1-\xi)$ and $B_{2}=B(1+\xi)$ with $|\xi|<1$. The elastic energy density now corresponds to

$$
f_{e}=\frac{1}{2} B(1-\xi \cos 2 \phi)\left(\frac{d \phi}{d x}\right)^{2} .
$$

In general, $\mathrm{SmC}^{*}$ liquid crystals possess a spontaneous polarisation $\boldsymbol{P}$ which, in terms of the model for $\mathrm{SmC}^{*}$ described here, can be written as a vector parallel to the vector $\boldsymbol{b}$,

$$
\boldsymbol{P}=P_{0} \boldsymbol{b}=P_{0}(-\sin \phi(x), \cos \phi(x), 0),
$$

for constant magnitude $P_{0}$. ( $P_{0}>0$ corresponds to the usual sign convention for positive polarisation.) The spontaneous polarisation now contributes to the total energy density via

$$
f_{p}=-\boldsymbol{P} \cdot \boldsymbol{E} .
$$

Further, the dielectric energy density for the ferroelectric can be expressed in terms of vectors $\boldsymbol{a}$ and $c$ as

$$
f_{d}=-\frac{1}{2} \epsilon_{0} \epsilon_{a}(\boldsymbol{a} \cdot \boldsymbol{E} \cos \theta+\boldsymbol{c} \cdot \boldsymbol{E} \sin \theta)^{2},
$$

where $\epsilon_{0}$ is the permittivity of free space and $\epsilon_{a}$ is the dielectric anisotropy. Therefore, the total overall energy density for our system is

$$
\begin{aligned}
\mathcal{F}= & f_{e}+f_{p}+f_{d} \\
= & \frac{1}{2} B(1-\xi \cos 2 \phi)\left(\frac{d \phi}{d x}\right)^{2}+P_{0}\left(E_{x} \sin \phi-E_{y} \cos \phi\right) \\
& -\frac{1}{2} \epsilon_{0} \epsilon_{a} \sin ^{2} \theta\left(E_{x} \cos \phi+E_{y} \sin \phi\right)^{2},
\end{aligned}
$$

where we have assumed that $\boldsymbol{E}=\left(E_{x}, E_{y}, 0\right)$ is parallel to the smectic layers. In the following sections we will consider an applied electric field either perpendicular or parallel to the domain wall. These 
configurations correspond to $E=E(1,0,0)$ or $E=E(0,1,0)$, respectively, where $E$ is the magnitude of the field.

\section{ELECTRIC FIELD PERPENDICULAR TO THE DOMAIN WALL}

Figure 2 illustrates the direction of the spontaneous polarisation $\boldsymbol{P}$ and director $c$ within a smectic layer under the influence of a field applied perpendicular to the domain wall. Recall that $c$ is the unit projection of the director $\boldsymbol{n}$ onto the smectic later. Therefore, the rotation of $\boldsymbol{c}$ within the $x y$-plane mirrors the twist behaviour of the director $\boldsymbol{n}$.

In this geometry $E_{x}=E, E_{y}=0$ and from Eq. (1) the total energy density is

$$
\mathcal{F}=\frac{1}{2} B(1-\xi \cos 2 \phi)\left(\frac{d \phi}{d x}\right)^{2}+P_{0} E \sin \phi-\frac{1}{2} \epsilon_{0} \epsilon_{a} E^{2} \cos ^{2} \phi \sin ^{2} \theta .
$$

Equilibrium director profiles within the smectic film correspond to minima of the total free energy. These profiles are derived from solutions of the Euler-Lagrange equations corresponding to Eq. (2), namely

$$
(1-\xi \cos 2 \phi) \phi^{\prime \prime}+\xi\left(\phi^{\prime}\right)^{2} \sin 2 \phi-\frac{P_{0} E}{B} \cos \phi-\frac{\epsilon_{0} \epsilon_{a} E^{2}}{B} \sin ^{2} \theta \sin \phi \cos \phi=0,
$$
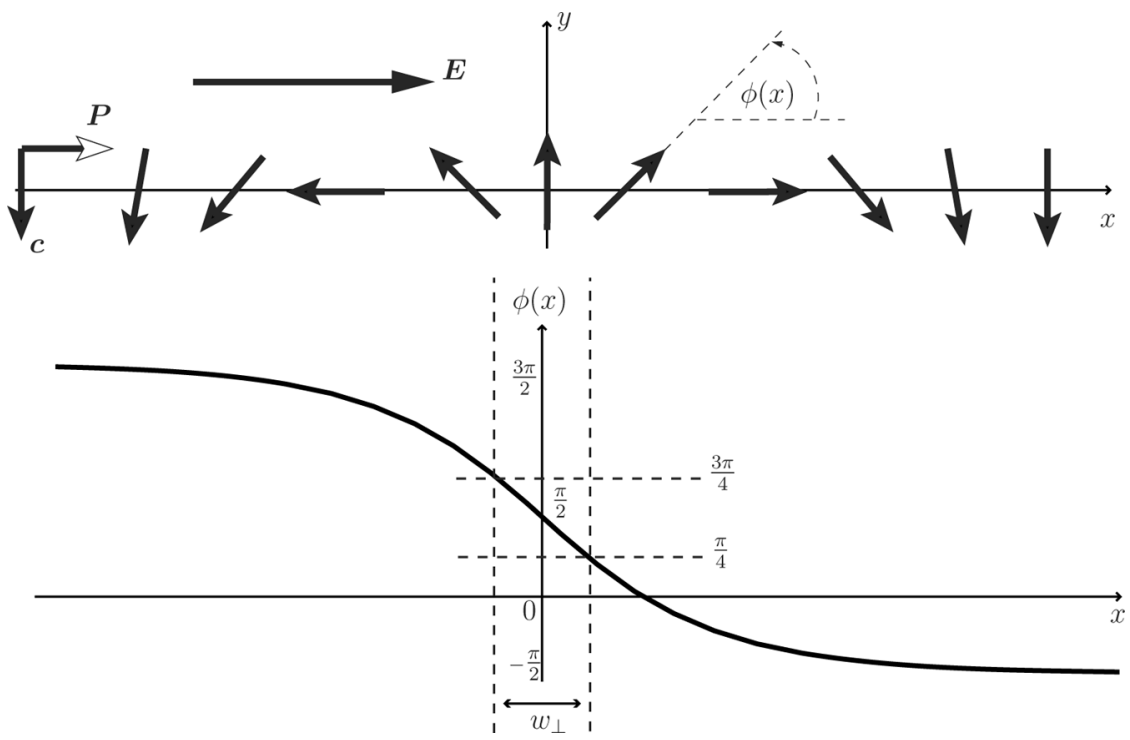

FIGURE $22 \pi$-rotation of the director $\boldsymbol{c}$ in the domain wall perpendicular to $\boldsymbol{E}$. 
where / denotes a derivative with respect to $x$. Domain wall solutions to Eq. (3) will now be determined. Such walls will be over an infinite domain in $x$ and centred at $x=0$, subject to the boundary conditions

$$
\phi \rightarrow \frac{3 \pi}{2} \text { as } x \rightarrow-\infty, \quad \phi \rightarrow-\frac{\pi}{2} \text { as } x \rightarrow \infty, \quad \phi^{\prime} \rightarrow 0 \text { as } x \rightarrow \pm \infty,
$$

so that we are seeking $2 \pi$-domain walls. As indicated in Figure 2 , the width of the domain wall, $w_{\perp}$, is defined to be the length over which the director twist changes from $\phi=\pi / 4$ to $3 \pi / 4$, i.e., one quarter of the total domain wall. Multiplying Eq. (3) by $\phi^{\prime}$, integrating over the width of the wall and employing the boundary conditions (4), we can derive a formula for the domain wall width, namely

$$
w_{\perp}=\sqrt{\frac{B}{2 P_{0} E}} \int_{\pi / 4}^{3 \pi / 4}\left(\frac{1-\xi \cos 2 \phi}{1+\sin \phi+\lambda \cos ^{2} \phi}\right)^{1 / 2} d \phi,
$$

where $\lambda=-\epsilon_{0} \epsilon_{a} E \sin ^{2} \theta /\left(2 P_{0}\right)$. Although it is an exact formula for the wall width, the integrand in Eq. (5) cannot be integrated analytically with respect to $\phi$. However, since $|\xi|<1$ it is possible to expand the integrand as a multivariate power series in $\xi$ and $\lambda$ whenever $|\lambda|<2+\sqrt{2}$, showing that

$$
w_{\perp} \simeq \sqrt{\frac{B}{2 P_{0} E}} \sum_{n=0}^{N} \sum_{m=0}^{N}\left(\begin{array}{c}
\frac{1}{2} \\
n
\end{array}\right)\left(\begin{array}{c}
-\frac{1}{2} \\
m
\end{array}\right)(-\xi)^{n} \lambda^{m} \int_{\pi / 4}^{3 \pi / 4} \frac{\cos ^{n} 2 \phi \cos ^{2 m} \phi}{(1+\sin \phi)^{m+1 / 2}} d \phi,
$$

where $N$ is the order of the expansion in both variables. (The width $w_{\perp}$ defined in Eq. (5) is equivalent to that given in Eq. (6) in the limit as $N \rightarrow \infty$.) Unlike Eq. (5), the integrals that appear in Eq. (6) can be evaluated analytically for any non-zero integers $m$ and $n$. Therefore, for $\left|\epsilon_{0} \epsilon_{a}\right| E \sin ^{2} \theta<(4+2 \sqrt{2}) P_{0}$ and a suitable choice of $N$, Eq. (6) provides an accurate approximate value for the domain wall width. (The full series is exact when $|\lambda|<2+\sqrt{2}$.)

\section{ELECTRIC FIELD PARALLEL TO THE DOMAIN WALL}

We now consider an electric field applied in a direction parallel to the domain wall, see Figure 3. From Eq. (1) the total energy density in this case (with $E_{x}=0, E_{y}=E$ ) is

$$
\mathcal{F}=\frac{1}{2} B(1-\xi \cos 2 \phi)\left(\frac{d \phi}{d x}\right)^{2}-P_{0} E \cos \phi-\frac{1}{2} \epsilon_{0} \epsilon_{a} E^{2} \sin ^{2} \phi \sin ^{2} \theta .
$$



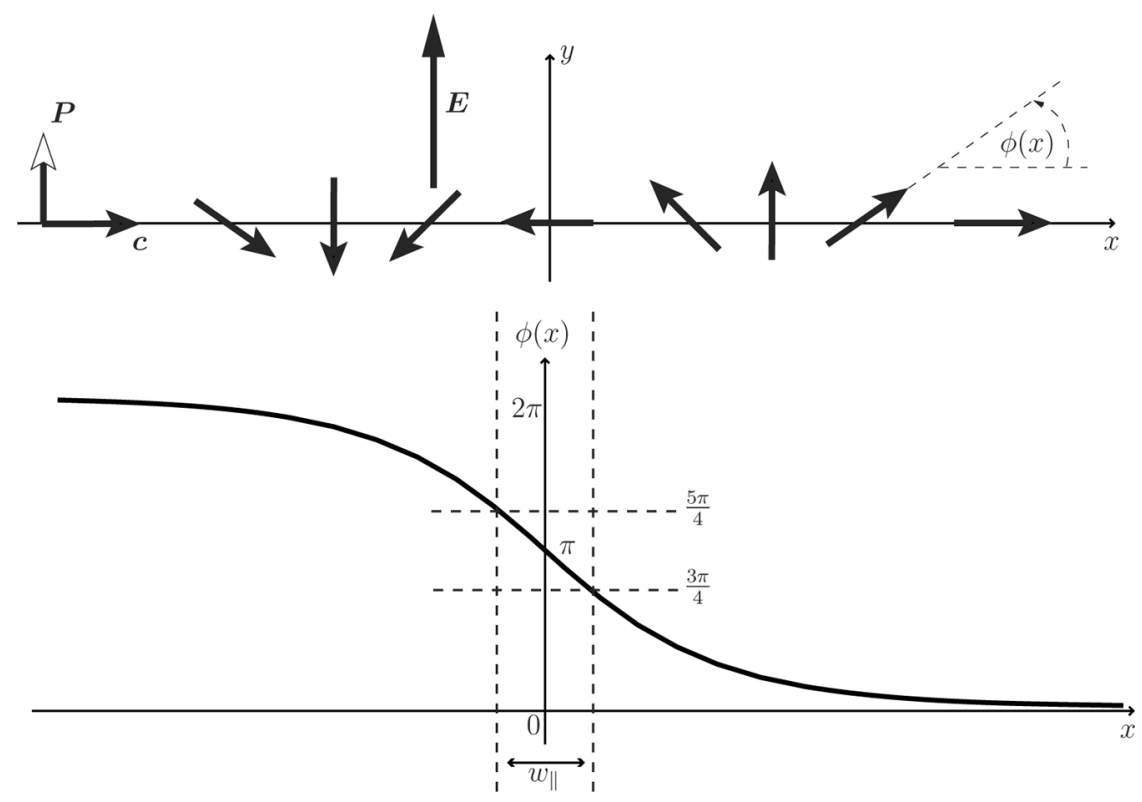

FIGURE $32 \pi$-rotation of the director $\boldsymbol{c}$ in the domain wall parallel to $\boldsymbol{E}$.

The corresponding Euler-Lagrange equations for minimisation of the free energy are

$$
(1-\xi \cos 2 \phi) \phi^{\prime \prime}+\xi\left(\phi^{\prime}\right)^{2} \sin 2 \phi-\frac{P_{0} E}{B} \sin \phi+\frac{\epsilon_{0} \epsilon_{a} E^{2}}{B} \sin ^{2} \theta \sin \phi \cos \phi=0 .
$$

The width of the domain wall, $w_{\|}$, can now be calculated via a procedure analogous to that employed in the previous section. We assume that far from the domain wall,

$$
\phi \rightarrow 2 \pi \text { as } x \rightarrow-\infty, \quad \phi \rightarrow 0 \text { as } x \rightarrow \infty, \quad \phi^{\prime} \rightarrow 0 \text { as } x \rightarrow \pm \infty .
$$

The wall width itself is defined as the region between $\phi=3 \pi / 4$ to $\phi=5 \pi / 4$, as shown in Figure 3. The exact integral formula and power series expansion obtained in this way are

$$
\begin{aligned}
w_{\|} & =\sqrt{\frac{B}{2 P_{0} E}} \int_{3 \pi / 4}^{5 \pi / 4}\left(\frac{1-\xi \cos 2 \phi}{1-\cos \phi+\lambda \sin ^{2} \phi}\right)^{1 / 2} d \phi \\
\simeq & \sqrt{\frac{B}{2 P_{0} E} \sum_{n=0}^{N} \sum_{m=0}^{N}\left(\begin{array}{c}
\frac{1}{2} \\
n
\end{array}\right)\left(\begin{array}{c}
-\frac{1}{2} \\
m
\end{array}\right)(-\xi)^{n} \lambda^{m}} \\
& \times \int_{3 \pi / 4}^{5 \pi / 4} \frac{\cos ^{n} 2 \phi \sin ^{2 m} \phi}{(1-\cos \phi)^{m+1 / 2}} d \phi,
\end{aligned}
$$


where Eq. (8) is valid for $|\xi|<1$ and $|\lambda|<2+\sqrt{2}$ (via a similar argument to the previous section). As before, this double series is exact for these ranges of $\xi$ and $\lambda$ when $N \rightarrow \infty$. Furthermore, the integrals in Eq. (8) can be calculated analytically.

\section{WALL WIDTH RATIO}

We can now combine the results of Eqs. (5), (7) to obtain an expression for the ratio of domain wall widths obtain for the two choices of electric field, namely

$$
\frac{w_{\perp}}{w_{\|}}=\frac{\int_{\pi / 4}^{3 \pi / 4}(1-\xi \cos 2 \phi) /\left(1+\sin \phi+\lambda \cos ^{2} \phi\right)^{1 / 2} d \phi}{\int_{3 \pi / 4}^{5 \pi / 4}(1-\xi \cos 2 \phi) /\left(1-\sin \phi+\lambda \sin ^{2} \phi\right)^{1 / 2} d \phi} .
$$

We recall that $\lambda=-\epsilon_{0} \epsilon_{a} E \sin ^{2} \theta /\left(2 P_{0}\right)$ and $\xi$ is a measure of the anisotropy between the two twist elastic constants. Alternatively, the

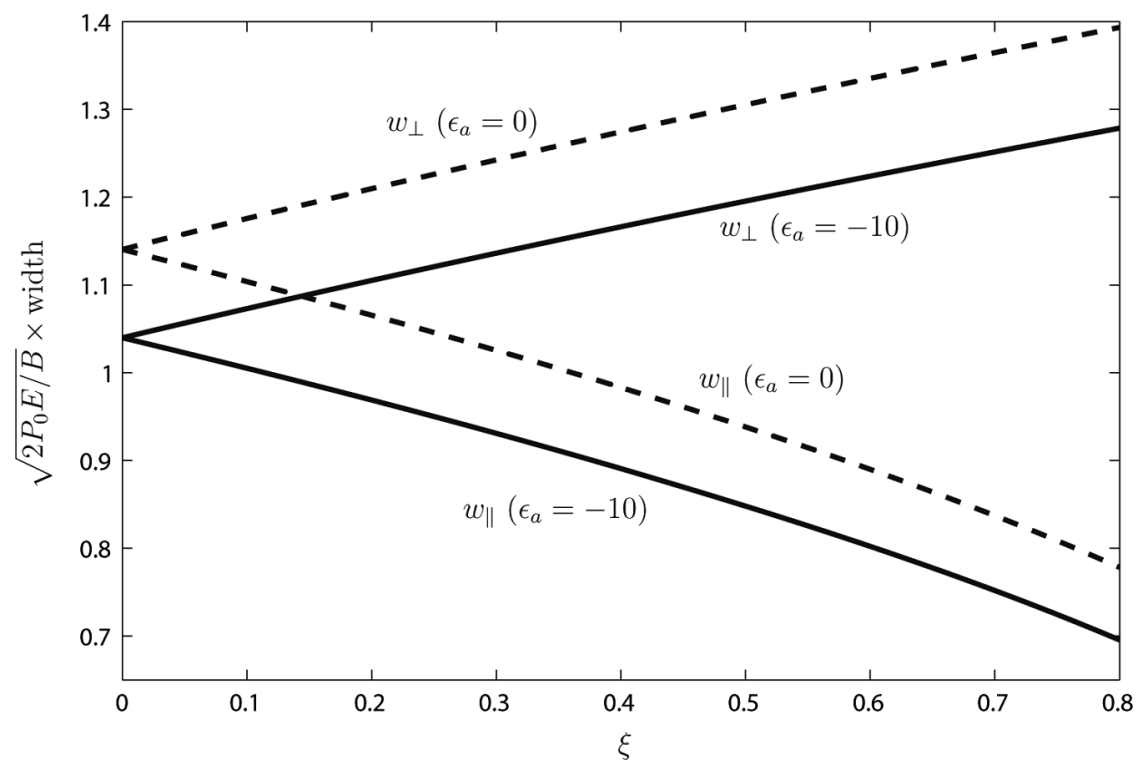

FIGURE 4 Wall widths for an electric field applied perpendicular, $w_{\perp}$, and parallel, $w_{\|}$, to the domain wall as the measure of elastic anisotropy varies. (---): dielectric anisotropy $\epsilon_{a}=0,(-)$ : dielectric anisotropy $\epsilon_{a}=-10$. 


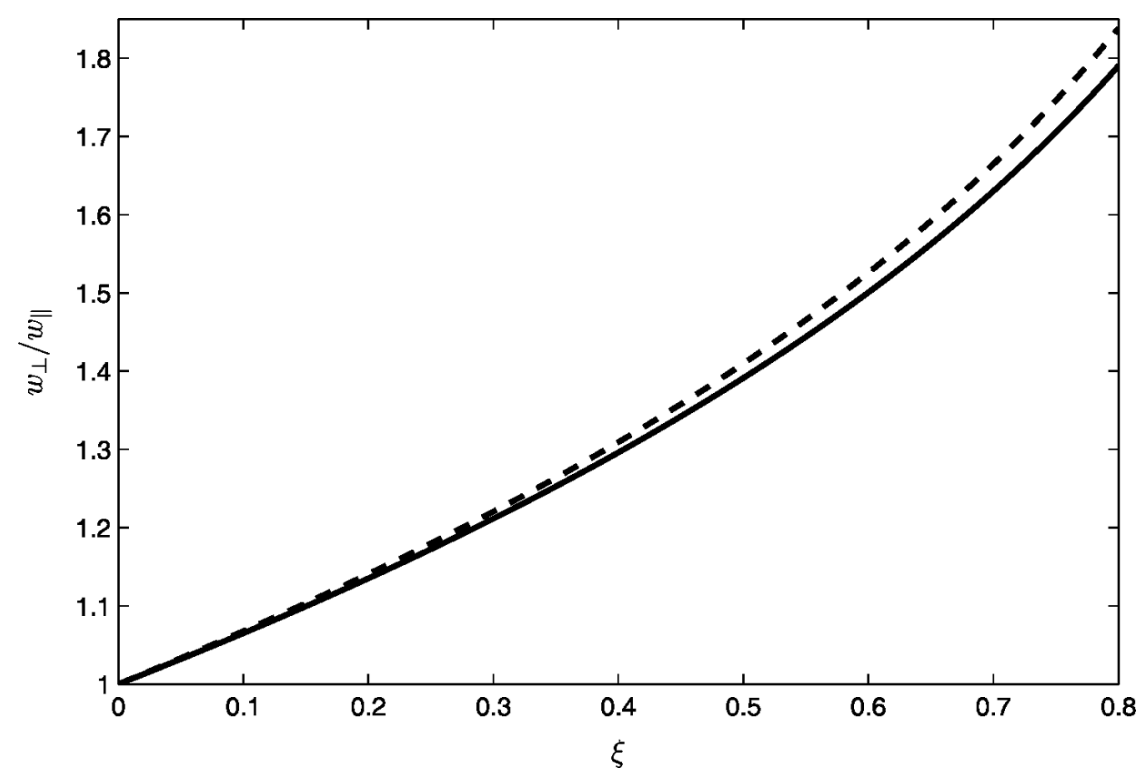

FIGURE 5 Ratio of wall widths $w_{\perp}$ and $w_{\|}$as anisotropy $\xi$ varies. (- - - ): dielectric anisotropy $\epsilon_{a}=0,(-)$ : dielectric anisotropy $\epsilon_{a}=-10$.

width ratio may be approximated as the ratio of the series derived in Eqs. (6) and (8).

For known material properties and a fixed electric field magnitude, the ratio given in Eq. (8) (or the equivalent approximate power series form) may be determined as a function of the anisotropy $\xi$ between elastic constants. Significantly, unlike previous analyses, the ratio (9) incorporates the influence of the dielectric properties of the ferroelectric material.

In Figures 4 and 5 we employ numerical quadrature to calculate the wall widths obtained in Eqs (5) and (7) for the following material parameters: $\theta=20^{\circ}, E=20 \mathrm{~V} \mathrm{~mm}^{-1}, P_{0}=100 \mu \mathrm{C} \mathrm{m}^{-2}, \epsilon_{0}=8.854 \times 10^{-12}$ $\mathrm{F} \mathrm{m}^{-1}$ (Stewart [4]). These parameter values correspond to $\lambda=2.135$. From Figure 4 we observe that the dielectric anisotropy reduces the size of both the perpendicular and parallel wall widths. However, in Figure 5 the influence of the dielectric anisotropy upon the width ratio is relatively small except when the elastic anisotropy is large. Figure 4 also indicates that the perpendicular width increases with elastic anisotropy while the parallel width decreases, therefore the ratio of wall widths increases significantly as elastic anisotropy increases. 


\section{REFERENCES}

[1] Swanson, B. D., Stragier, H., Tweet, D. J., \& Sorensen, J. B. (1989). Phys. Rev. Lett., $62,909$.

[2] Candel, V. \& Galerne, Y. (1993). Liq. Cryst., 15, 541.

[3] Leslie, F. M., Stewart, I. W., \& Nakagawa, M. (1991). Mol. Cryst. Liq. Cryst., 198, 443.

[4] Stewart, I. W. (2004). The Static and Dynamic Continuum Theory of Liquid Crystals, Taylor and Francis: London and New York. 